\title{
Successful Pregnancy in a Patient with Long-Standing Acromegaly - a Case Report
}

\author{
Maximilian Cosma Gliga1', Maria Ionela Pașcanu1,2, Camelia Gliga ${ }^{1,3}$, Zsuzsanna Réti² \\ 1 Department of Endocrinology, Mureș County Clinical Hospital, Târgu Mureș, Romania \\ 2 Department of Endocrinology, "George Emil Palade” University of Medicine, Pharmacy, Science and Technology, Târgu Mureș, Romania \\ 3 Department of Histology, "George Emil Palade” University of Medicine, Pharmacy, Science and Technology, Târgu Mureș, Romania
}

\section{CORRESPONDENCE}

Maximilian Cosma Gliga

Piața Victoriei nr. 19/2

540029 Târgu Mureș, Romania

Tel: +40741528161

E-mail: max_mg_tenn@yahoo.com

\section{ARTICLE HISTORY}

Received: June 1, 2020

Accepted: September 3, 2020
Maria Ionela Pașcanu • Str. Gheorghe Marinescu nr. 38, 540139 Târgu Mureș, Romania. Tel: +40 265215 551, E-mail: iopascanu@gmail.com

Camelia Gliga • Str. Gheorghe Marinescu nr. 38 540139 Târgu Mureş, Romania. Tel: +40 265215 551, E-mail: camelia.gliga@umfst.ro

Zsuzsanna Réti • Str. Gheorghe Marinescu nr. 38, 540139 Târgu Mureş, Romania. Tel: +40 265215551 E-mail: zsuzsanna.reti@umfst.ro

\section{ABSTRACT}

Introduction: Acromegaly is a rare endocrine disorder of the growth hormone (GH)-insulin-like growth factor 1 (IGF1) metabolism that can affect women of fertile age. Although uncommon, pregnancies in acromegalic women can occur, with data regarding the management of these cases being very limited, mostly consisting of case reports. Case Presentation: We present the case of an acromegalic woman, first diagnosed at the age of 22, after the surgical resection of a pituitary mass. Throughout the evolution, she received conventional radiotherapy and has been treated with somatostatin receptor ligands (SRLs), dopamine agonists (DAs), and GH-blockers. At the age of 37, the patient decided to become pregnant while she was on Pegvisomant and DA therapy. The treatment was stopped, and the patient became pregnant at the age of 38. Tumor size and IGF-1 values have remained stable throughout the pregnancy, and no complications occurred. A healthy child with normal birth weight was delivered on term through Cesarean section. Conclusions: Managing pregnant women with acromegaly is challenging because of the little available data regarding the safety of medical treatment and a high interindividual variability of GH-IGF-1 evolution during this period. The particularity of our case was a patient with a long history of acromegaly, who had an uneventful pregnancy despite stopping all medical treatment.

Keywords: acromegaly, pregnancy, treatment

\section{INTRODUCTION}

Acromegaly is a rare disorder, characterized by increased production of growthhormone (GH) by the anterior pituitary gland, leading to increased blood levels of insulin-like growth factor 1 (IGF-1), the hormone responsible for the majority of specific physio-pathological changes that occur in this syndrome. In acromegalic female patients of reproductive age, fertility is frequently impaired. Although rare, pregnancies can occur and are becoming more common in recent years due to an improvement in the therapeutic management of these patients. Current available data regarding pregnant women with acromegaly are limited, mostly composed of case reports, series of cases, and a few retrospective studies. ${ }^{1,2}$ 
The hormonal balance of the somatotropic axis during physiological pregnancy follows a dynamic course, which is characterized by a gradual decrease of pituitary GH (native $\mathrm{GH}, \mathrm{GH}-\mathrm{N}$ ), followed in the last two trimesters by rising levels of placental GH (variant GH, GH-V). Both GH-V and IGF-1 reach a peak around the last weeks of pregnancy, the latter being at least two times higher on average than before pregnancy. Another essential characteristic of the GH-IGF-1 axis during pregnancy is an increased resistance to GH due to high estrogen levels. ${ }^{3,4}$

In pregnant patients with acromegaly, $\mathrm{GH}-\mathrm{N}$ levels produced by the pituitary adenoma remain high because the secretion is mostly autonomous and will not respond to negative feedback from increased levels of IGF-1. Because of the previously mentioned estrogen-related GH-resistance, the majority of acromegalic patients are expected to maintain a stable, mild evolution or even a slight improvement during pregnancy. ${ }^{4}$ However, all these mechanisms present high inter-individual variability and tumor growth may occur, leading to optical disturbances and headache. Therefore, a careful observation of these patients is essential in order to achieve the best therapeutic outcomes for both the mother and the fetus. ${ }^{5,6}$

\section{CASE REPORT}

We present the case of a 22 -year-old female patient, Caucasian, from Mureș County, Romania, first admitted to the Endocrinology Clinic in 2000 with the following symptoms: 1-year history of dull, generalized headache, tiredness, acral enlargement, weight gain, coarsening and enlargement of facial features, associated with 12 months of amenorrhea. The clinical examination revealed a BMI of $24.2 \mathrm{~kg} / \mathrm{m}^{2}$, excessive body hair (Ferriman Gallwey scale $14 \mathrm{pts}$ ), bilateral galactorrhea, and no visual impairment or nasal discharge. The initial laboratory tests showed mild hyperprolactinemia (PRL: $78.15 \mathrm{ng} / \mathrm{mL}$ ), LH: $2.24 \mathrm{mIU} / \mathrm{mL}$, FSH: $4 \mathrm{mIU} / \mathrm{mL}$, estradiol $30.05 \mathrm{pg} / \mathrm{mL}$ (NV: 9-30), hyperandrogenism (testosterone $0.67 \mathrm{ng} / \mathrm{mL}, \mathrm{NV}$ : 0.09-0.56), normocorticism (8 AM cortisol $15.75 \mu \mathrm{g} / \mathrm{dL}$ ), and euthyroid status (fT4 $11.97 \mathrm{pmol} / \mathrm{L})$. The computed tomography $(\mathrm{CT})$ scan revealed a $13 \times 18 \mathrm{~mm}$ pituitary tumor reaching the cavernous sinuses. The patient was referred to a regional neurosurgery hospital, where transsphenoidal ablation of the tumor was performed. The diagnosis of acromegaly was established based on the immunohistochemistry examination of the tumor, which showed a positive result for $\mathrm{GH}$, and negative for PRL, LH, and FSH.

A few months later, in 2001, on the next follow-up visit after the surgical treatment, the patient presented with- out significant clinical signs and symptoms of recurrence. Magnetic resonance imaging (MRI) of the pituitary region performed in 2002 showed a tumoral mass of $12 \times 14 \mathrm{~mm}$, with sphenoidal extension of $16 \mathrm{~mm}$, while the oral glucose tolerance tests (OGTT) confirmed the progression of active acromegaly (Table 1 ).

During the following years, the symptoms have progressively worsened, with headache and retrobulbar pain. In 2005, the patient presented severe progressive headache and visual disturbances, the MRI examination revealing significant tumor growth (Figure 1).

Considering the tumoral relapse and the worsening of disease control, surgical reintervention was performed for a debulking procedure. Four months after the surgery, the patient underwent conventional radiotherapy (50 Gy) and medical treatment with a somatostatin receptor ligand (SRL), lanreotide was initiated. For the next few years, from 2005 until 2009, the patient had a relatively stable evolution, with increasing doses of lanreotide required because of poor response to medication, IGF-1 values and GH levels during the OGTT remaining high (Figure 2 and Table 1).

Considering the resistance to SRL and the consistently high IGF-1 and GH levels, treatment with pegvisomant in loading dose of $80 \mathrm{mg}$, with $10 \mathrm{mg} /$ day continuation, was initiated. The pituitary MRI performed in 2009 showed a tumoral remnant of $15 \times 18 \times 16 \mathrm{~mm}$ (Figure 3 ).

Under pegvisomant, IGF-1 values quickly normalized, with levels of $289 \mathrm{ng} / \mathrm{mL}$ after one month and $150 \mathrm{ng} / \mathrm{mL}$ after three months, showing a good response to treatment. From 2011 until 2014, the patient underwent treatment with pegvisomant $10 \mathrm{mg} /$ day and cabergoline $1 \mathrm{mg} /$ week. Under treatment she had normal periods, and MRI exams revealed no changes in the tumor size while IGF-1 values remained stable and in normal range during this period.

In 2014, at the age of 37 years, she decided to become pregnant, therefore pegvisomant treatment was stopped, and cabergoline was continued at low doses of $0.5 \mathrm{mg} /$ week. A genetic testing for familial isolated pituitary adenoma was conducted, the results confirming no patho-

TABLE 1. Oral glucose tolerance test performed across an 8-year time span

\begin{tabular}{llllll}
\hline & Time $(\mathbf{m i n})$ & $\mathbf{0}$ & $\mathbf{3 0}$ & $\mathbf{6 0}$ & $\mathbf{1 2 0}$ \\
\hline 2001 & $\mathrm{GH}(\mathrm{ng} / \mathrm{mL})$ & 7.1 & 5.6 & 5.7 & 6.9 \\
2002 & $\mathrm{GH}(\mathrm{ng} / \mathrm{mL})$ & 24.8 & 23 & 19.4 & 20.3 \\
2008 & $\mathrm{GH}(\mathrm{ng} / \mathrm{mL})$ & 18.8 & 18.3 & 14.3 & 12.3 \\
2009 & $\mathrm{GH}(\mathrm{ng} / \mathrm{mL})$ & 15.2 & 15.4 & 11.6 & 11.9 \\
\hline
\end{tabular}



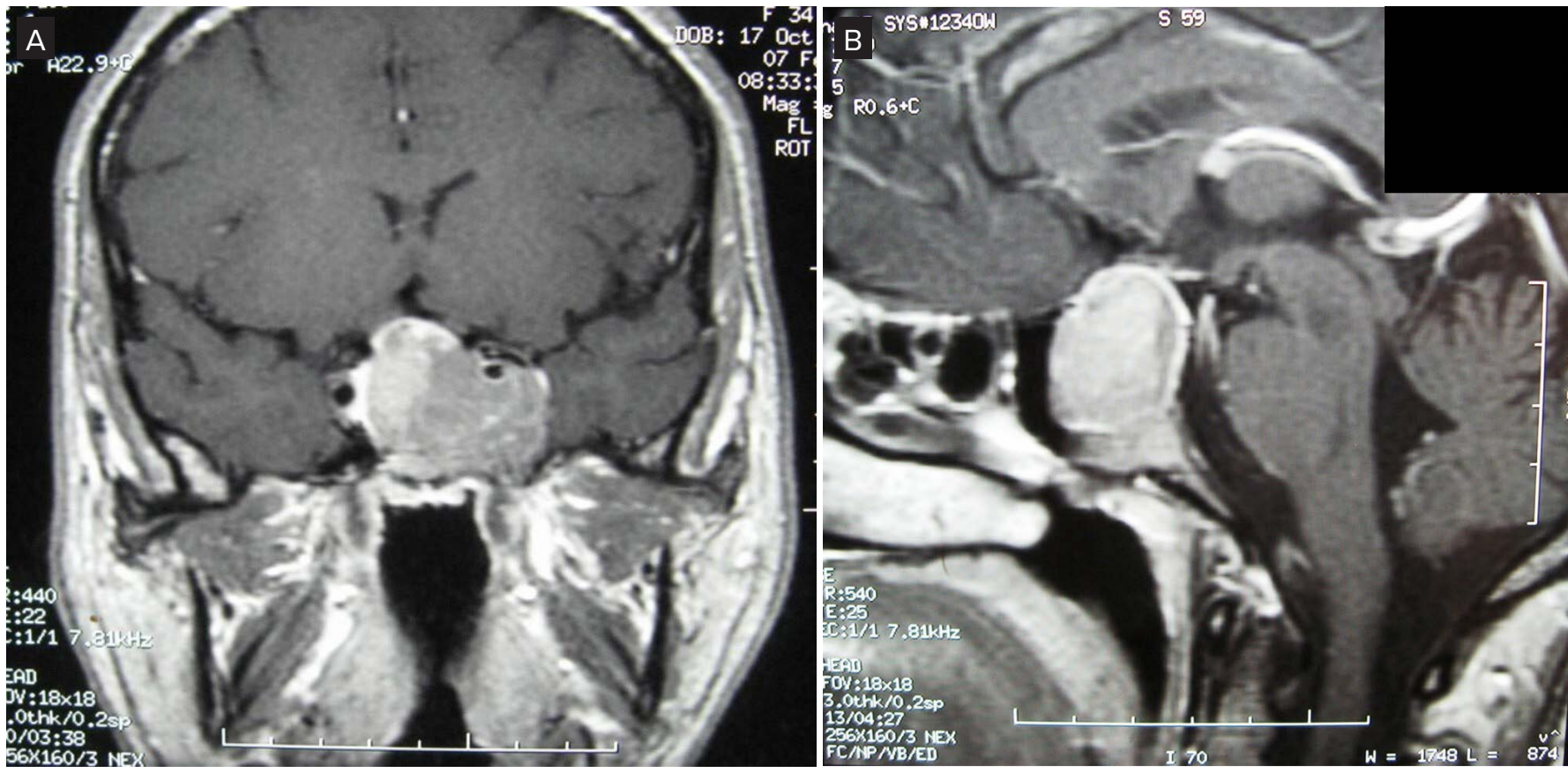

FIGURE 1. Pituitary magnetic resonance imaging. A - Coronal section showing a $38 \times 21 \times 27$ mm formation with supra- and parasellar extension mainly to the left and in the sphenoid sinus, elevating the optic chiasm. The left internal carotid artery is surrounded by the parasellar tumor extension. B - Sagittal section illustrating the pituitary tumor

genic mutations in the AIP gene. MRI follow-up showed no significant growth in tumor size, and the control of acromegaly disease continued to be optimal, with IGF-1 values in normal ranges. The $8 \mathrm{AM}$ cortisol levels showed a slight decrease, with values of $8.1 \mu \mathrm{g} / \mathrm{dL}$ in 2015 , but no clinical signs of central adrenal insufficiency were noted.
At the age of 38 , the patient became pregnant after uterine insemination, and the cabergoline treatment was stopped. During the course of the pregnancy, no tumoral growth and symptoms were reported, and IGF-1 values remained stable. The patient gave birth to a healthy baby with normal birth weight, delivered on term through $\mathrm{Ce}$ -

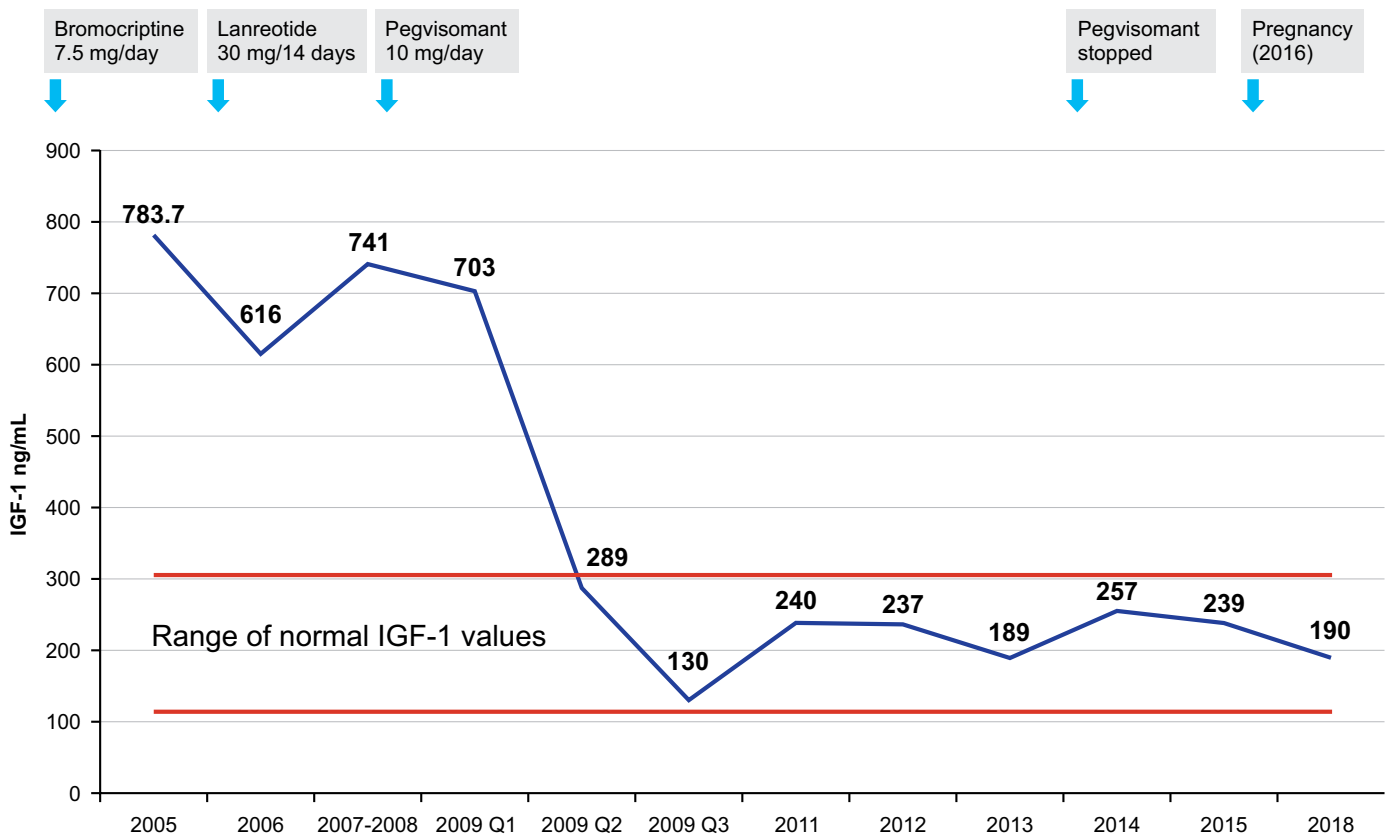

FIGURE 2. Evolution of IGF-1 levels between 2005 and 2009 

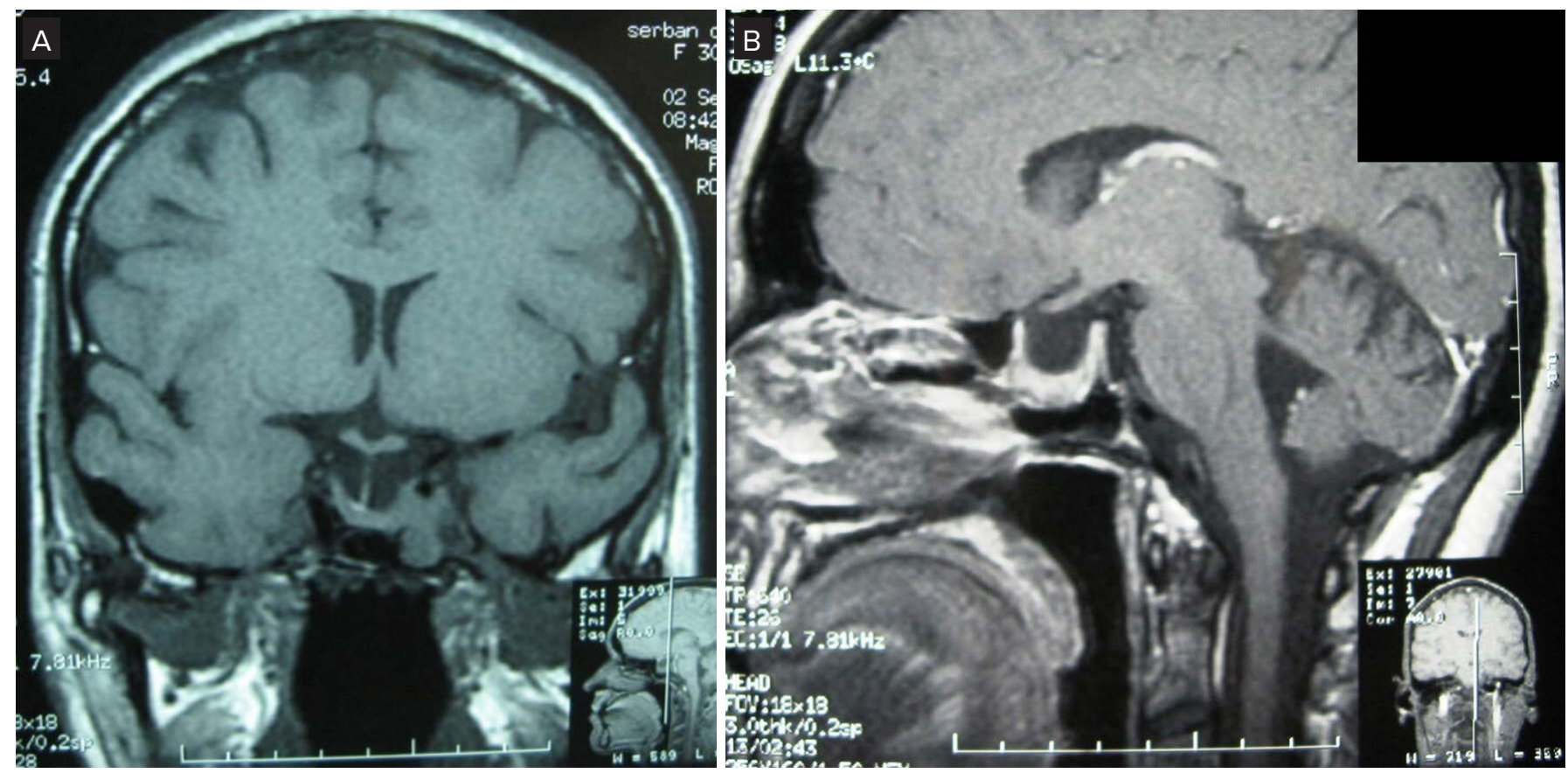

FIGURE 3. Pituitary MRI examination. A - Coronal section showing a tumoral remnant of $15 \times 7 \times 10 \mathrm{~mm}$, in the proximity of the left cavernous sinus; $\mathbf{B}$ - Sagittal section showing the same findings

sarean section. The patient breastfed her baby for two years after giving birth, without any issues or complications. At the 1-year follow-up after giving birth, the IGF-1 values remained normal, and the OGTT showed suppressible GH with nadir value below $1 \mathrm{ng} / \mathrm{mL}$. The last MRI investigations revealed a similar size of the tumoral remnant with no significant growth since the last imaging examination. Other hormonal tests showed normal estradiol, FSH, and LH levels, euthyroid state and normocorticism, and mildly increased levels of prolactin (PRL $28.21 \mathrm{ng} / \mathrm{mL}$ ).

The patient agreed to the publication of her data, and the institution where the patient had been admitted, approved the publication of the case.

\section{DISCUSSIONS}

The impact of acromegaly on pregnancies is a debatable subject of increasing interest. There is currently little available data regarding the optimal management and therapy for these patients, or the safety profile of various treatment options.

First of all, clinicals should consider the possibility of tumor growth that could further lead to neurological or visual complications. There are many mechanisms responsible for this process, from the estrogen-related physiological hyperplasia of the pituitary gland to pituitary apoplexy or growth secondary to discontinuation of medical therapy due to safety issues. Despite this, most data from case reports of pregnant acromegalic women show that very few patients develop significant tumor enlargement that would lead to visual problems, headaches being more frequent. ${ }^{7-9}$ The risk for tumor growth is further reduced for patients with microadenomas or who underwent surgery in the past. ${ }^{5}$ In our patient, who went through two surgeries and conventional radiotherapy, the evolution of tumor size had a similar pattern to most documented cases so far, with no tumor growth-related complications throughout her pregnancy. There is a concern that, in theory, breastfeeding could lead to an increase in the pituitary tumor due to stimulus from the suckling. However, previous reports indicated that there is no correlation between breastfeeding and tumor enlargement. Therefore, current guidelines indicate that there is no reason to advise against breastfeeding in acromegalic patients who presented an uneventful pregnancy.6,10,11 Our patient breastfed her daughter without any noticeable complications.

The majority of treatment options for patients with acromegaly, such as surgery, radiotherapy, medical treatment with dopamine agonists (DAs), SRLs, or pegvisomant, could pose risks for the fetus and the mother. Data regarding their safety during pregnancy are still very limited and unclear. Surgical treatment should be avoided because of the potential risks related to anesthesia and increased fetal morbidity, and should be performed, if absolutely neces- 
sary, in the second trimester. ${ }^{12}$ Radiotherapy is conventionally contraindicated in all pregnant patients with GH-secreting tumors. ${ }^{13}$ Current recommendations regarding DAs indicate that these should generally be discontinued when pregnancy is confirmed. However, many studies on large numbers of pregnant patients with prolactinomas have reported that bromocriptine or cabergoline could safely be administered. ${ }^{14}$ Most reports indicate no adverse effects for the mother or fetus after DA administration during pregnancy. However, in one study, a small number of newborns born from DA-treated mothers were reported with low birth weight for their gestational age, but no malformations were noted. ${ }^{15}$ Our patient stopped the DA treatment when uterine insemination was performed. SRLs are the first line of medical treatment in acromegaly. They are known to cross the placental barrier, and there are currently no studies to confirm the safety of their use in pregnant acromegalic women. Data regarding exposure of pregnant women to SRLs are limited to a few case reports. The current consensus suggests that they should be stopped once pregnancy is confirmed, and in case a patient wants to remain pregnant, switching to a short-acting SRL until pregnancy confirmation is indicated. ${ }^{6}$ In our case, SRL resistance with lack of response of the GH-IGF1 axis even to high doses of lanreotide was noticed, therefore treatment was stopped a few years prior to becoming pregnant.

The GH-blocker pegvisomant is an attractive therapeutic option for acromegalic patients, causing considerable reduction of IGF-1 levels and thus leading to less acromegalyrelated complications. Nevertheless, data regarding its use during pregnancy and the risks of fetal exposure are limited, with only three case reports of patients who were under pegvisomant treatment when pregnancy was confirmed. In one case, gestational diabetes, pituitary gland growth, and visual disturbance occurred, but the child was safely delivered at 32 weeks, while in the other cases no complications or malformations related to pegvisomant exposure were noted. ${ }^{6}, 16$ Our presented case had a good response to pegvisomant treatment during the three years prior to deciding on becoming pregnant, with no tumor growth occurring and significant improvements in IGF-1 levels. Treatment was stopped after the patient decided on trying to become pregnant, as recommended by the latest guidelines.

Pregnant acromegalic patients should also be screened for gestational diabetes or hypertension, either of which could develop or worsen because of the impaired $\mathrm{GH}$ IGF1 metabolism in acromegaly. ${ }^{16}$ In our case, the metabolic control of the GH-IGF1 axis was optimal, and no glucose metabolism disorders or high blood pressure was observed during the course of the pregnancy.
The particularity of our case was an uneventful evolution of pregnancy in a patient with a long history of acromegaly, diagnosed 15 years before giving birth. She underwent two surgeries and conventional radiotherapy, yet did not develop hypogonadism. During the evolution of the disease, she was treated with a first-generation SRL (lanreotide), and although resistant to this therapeutic option, she was eventually well controlled with GH-blocker therapy (pegvisomant). The patient was under pegvisomant and DA treatment prior to deciding on becoming pregnant. The GH-blocker was interrupted, and the DA dose was reduced until pregnancy confirmation. No tumor growth, metabolic imbalances, or other complications occurred despite the interruption of all treatment throughout the pregnancy.

\section{CONCLUSIONS}

Pregnancy in acromegaly is a rare occurrence, considering the average age of onset for the disease and its consequences on fertility. However, it usually carries a good prognosis for the fetus and the mother if careful monitoring and optimal biochemical control is maintained. There is little evidence regarding the safety profile of acromegaly-specific medical treatment, and general consensus suggests it should be interrupted when pregnancy is confirmed and only be used in the rare cases where symptomatic tumor growth occurs and the estimated benefits outweigh the risks. Larger scale studies and data derived from clinical trials are required to determine the optimal management of pregnant acromegalic patients and the safety profile of the available medical treatment options.

\section{ACKNOWLEDGEMENT}

We thank Pfizer for the support and funding of this research.

\section{CONFLICT OF INTEREST}

Nothing to declare.

\section{REFERENCES}

1. Abucham J, Bronstein MD, Dias ML. Management of Endocrine Disease: Acromegaly and pregnancy: a contemporary review. Eur J Endocrinol. 2017;177: R1-R12.

2. Muhammad A, Neggers SJ, Van der Lely AJ. Pregnancy and acromegaly Pituitary. 2017;20:179-184

3. Braunstein, G. Endocrine changes in pregnancy. In: Melmed, S. (ed.), Williams Text Book of Endocrinology. Philadelphia: Saunders, 2011; p. 819-832.

4. Fuglsang J, Ovesen P. Aspects of placental growth hormone physiology. Growth Horm IGF. 2006;16:67-85. 
5. Cozzi R, Attanasio R, Barausse M. Pregnancy in acromegaly: a one center experience. Eur J Endocrinol. 2006:155:279-284.

6. Chanson P, Vialon M, Caron P. An update on clinical care for pregnant women with acromegaly. Expert Rev Endocrinol Metab. 2019;14:85-96.

7. Cheng S, Grasso L, Martinez-Orozco JA, et al. Pregnancy in acromegaly: experience from two referral centers and systematic review of the literature. Clin Endocrinol (Oxf). 2012;76:264-271.

8. Guven S, Durukan T, Berker M, et al. A case of acromegaly in pregnancy: concomitant transsphenoidal adenomectomy and cesarean section. J Matern Fetal Neonatal Med. 2006:19:69-71.

9. Kupersmith MJ, Rosenberg C, Kleinberg D. Visual loss in pregnant women with pituitary adenomas. Ann Intern Med. 1994;121:473-477.

10. Colao A, Merola B, Ferone D, et al. Acromegaly. J Clin Endocrinol Metab. 1997;82:2777-2781
11. Obuobie K, Mullik V, Jones C, et al. McCune-Albright syndrome: growth hormone dynamics in pregnancy. J Clin Endocrinol Metab. 2001:86:24562458.

12. Chowdhury T, Chowdhury M, Schaller B, et al. Perioperative considerations for neurosurgical procedures in the gravid patient: Continuing Professional Development. Can J Anaesth. 2013;60:1139-1155.

13. Hierl T, Ziegler R, Kasperk C. Pregnancy in persistent acromegaly. Clin Endocrinol (Oxf). 2000;53:262-263.

14. Molitch ME. Endocrinology in pregnancy: management of the pregnant patient with a prolactinoma. Eur J Endocrinol. 2015;172:R205-R213.

15. Cundy T, Grundy EN, Melville H, et al. Bromocriptine treatment of acromegaly following spontaneous conception. Fertil Steril. 1984:42:134-136.

16. Brian SR, Bidlingmaier M, Wajnrajch MP, et al. Treatment of acromegaly with pegvisomant during pregnancy: maternal and fetal effects. J Clin Endocrinol Metab. 2007;92:3374-3377. 